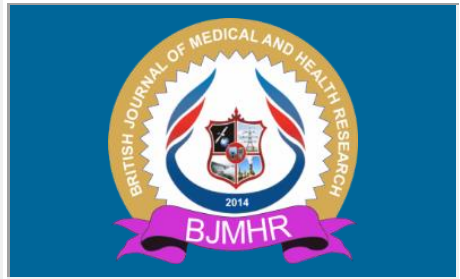

\title{
BJMHR
}

British Journal of Medical and Health Research

Journal home page: www.bjmhr.com

\section{Ameliorative Effects of Some Plant Seeds against side effects of antiobesity drug on Rats}

\section{Ola M. Abd-elatty, Heba M. El-ash, Heba A. El-dash, Abdelkarim M. Abd-ellatif* \\ Zoology Department - Faculty of Science - Fayoum UniversityZoology Department, Faculty of Science, Fayoum University, Egypt.}

\section{ABSTRACT}

This study aimed to investigate the antiobesity effects of lupin (L) and/or purslane (P) on obese female rats, 90 female albino rats divided into 9 groups. GPI (control non obese) fed on normal diet through 18 weeks and obesity groups were induced by feeding on a high-fat-diet during 3 months. At $310 \pm 10 \mathrm{~g}$, eighteen obese female rats were divided into eight groups and fed a high fat diet (HFD) or HFD containing either 30\% lupin seed powder and / or 30\% purslane seed powder or both for 6 weeks as following: GPII (Control obese), GPIV (L), GPV (P), GPVI $(\mathrm{L}+\mathrm{P})$, GPVII $(\mathrm{St}+\mathrm{L})$, GPVIII $(\mathrm{St}+\mathrm{P})$, GPIX $(\mathrm{St}+\mathrm{L}+\mathrm{P})$. Biochemical parameters as AST, ALT, $\mathrm{LDH}$, and blood urea nitrogen (BUN) and serum creatinine beside histopathological investigation for some vital organs were done. HFD induced obesity in GPII and elevated ALT, AST, LDH, BUN and creatinine. Also starvex drug showed clear anti-obesity effect on obese female rats more than lupin or purslane seeds powders but starvex significantly elevated ALT, AST, LDH, BUN creatinine coincides with the recorded histopathological lesions in the studied paraffin sections. Treatments with lupin and/or purslane seeds powders improve the biochemical elevation and histopathological observations in both control obese and starvex rats indicating their effect against toxicity. Starvex toxicity in obese female rats could be reduced by adding lupin seed and/or purslane.

Keywords: Obesity, Starvex, Lupin, Purslane, HFD. 


\section{INTRODUCTION}

Obesity is a metabolic disorder which occurs due to an energy imbalance between an increased ratio of caloric intake and a decreased ratio of energy expenditure (Sharpe et al. ${ }^{1}$. Obesity may begin several risk complications such as diabetes, hypertension, cardiovascular diseases, and some types of cancer (Ebrahimzadeh Attari et al. ${ }^{2}$. Obesity is a multifactorial disease that develops from a complex of interactions among genetic, metabolic, behavioral, as well as environmental factors $\left(\mathrm{WHO}^{3}\right.$.

Drugs approved for treatment of obesity are only for a short term, whereas a long-term is needed. Antiobesity drugs usually work by suppressing appetite, inhibiting fat absorption, or increasing energy consumption and thermogenesis (Cheung et al ${ }^{4}$. Most of the studies indicate fatty stool and decrease in absorption of fat soluble vitamin. However, most of the anti-obesity drugs are found to possess major side-effects with such as palpitation, tremors, hyperactive reflexes, hypertensive crisis with adrenergic drugs, monoamine, serotonergic drugs (Habibuddin and Humaira, ${ }^{5}$. However, most of the antiobesity drugs that were approved and marketed have now been withdrawn due to serious adverse effects. The naturopathic treatment for obesity has been explored extensively since ancient times and gaining momentum in the present scenario. Traditional medicinal plants and their active phytoconstituents have been used for the treatment of obesity and their associated secondary complications. The World Health Organization declared that about $80 \%$ of the world population is essentially dependent on herbal traditional drugs which came from the virtue of pragmatic knowledge (Ekor, ${ }^{6}$. Natural products are promising alternative sources due to their effective biological activities and potentially less severe side effects (Fu, et al., ${ }^{7}$.

Also, an array of natural products including plant crude extracts and their bioactive factors have been reported to induce weight loss, prevent diet induced obesity and allied ailments (Bhutani et al., ${ }^{8}$. The possible mechanisms through which such bioactive factors exert their antiobesity effects include appetite regulation, interfering in lipid digestion and absorption, fatty acid oxidation, regulating lipogenesis lypolysis and adipogenesis (Yang et al. ${ }^{9}$.

It was reported that lupin seeds have various nutritional and functional benefits, they are rich in proteins (about 50\%) and various fibers (Elbandy and Rho, ${ }^{10}$. Similarly, purslane has many bioactive compounds with multiple pharmacological and medical properties. It can presumably be considered, as food supplement and treatment of obesity (El-Zawahry et al., ${ }^{11}$. Rats are frequently used as animal models for studying adverse effects of obesity (Iossa et al., ${ }^{12}$. The obese animal model used in the experiment showed obviously increased body weight gain and lipid accumulation as reported before the anti-obesity treatment (Preston et al., ${ }^{13}$. 


\section{MATERIALS AND METHOD}

\section{Plant material preparations:}

\section{Lupines seed:}

Dried lupine seeds were purchased from the local market in Fayoum, (Egypt). The seeds were washed, dried at $37^{\circ} \mathrm{C}$ for $24 \mathrm{~h}$, and finely grinded (powdered) by mechanical grinder. Thirty grams of the termis powder is added and mixed with $100 \mathrm{~g}$ of normal diet.

Purslane seed were purchased from the local market in Fayoum, (Egypt). The seeds were washed and air dried at room temperature at $37^{\circ} \mathrm{C}$ for $24 \mathrm{~h}$, and finely grinded (powdered) by mechanical grinder. Thirty grams of the purslane powder is added and mixed with $100 \mathrm{~g}$ of normal diet.

Both lupin and purslane seeds were identified and verified by a botanist of Department of Botany, Faculty of Science, Fayoum University.

\section{Starvex: called Starvex BLUE}

Works on weight loss by locking and discouraging excessive appetite. It also increases the rate of fat burning and depletion stored in the body. Slimming abdomen, buttocks, shaping and sculpting. Each capsule contain: Aloe Vera 150mg, Garcinia cambogia 100mg and Hoodia Gordonii 100mg

\section{Daily dose and precaution}

One capsule per day before breakfast for a full hour or as directed by Dr. Pregnant and lactating until one year of breastfeeding or less than 14 years old.

\section{Normal and High fat diet compositions}

Beef thallow 29.5 g, Casein 22.0 g, Starch 23.0 g, Cellulose 17.9 g, L Cystiene 4.0 g, Choline chloride $0.3 \mathrm{~g}$, Vitamin mixture $1.8 \mathrm{~g}$, Mineral mixture $1.5 \mathrm{~g}$ were used $47 \%$ soybeans, soybeans seed, Limestone (Ca Co3), D. L methionine, choline chloride 1987, Sod. Bicarbonate, $\mathrm{NaCl}$, growth activator 2903, yellow corn, mixture of vitamins and minerals 4273 , calcium monophosphate,

\section{Animal and Experimental design}

Rats were divided into nine equal groups randomly. Rats in first group were fed normal diet and water ad libitum during the course of the experimental period (normal control) (18 weeks). Other groups of rats were first made obese by giving high fat diet (HFD) and water ad libitum for 12 weeks duration and then after divided and treated orally with different studied materials (starvex, lupin and purslane) along with continuation of high fat diet (obese treated) for 6 weeks A total of 90 healthy female albino rats with an average weight of $200 \pm 30$ grams were selected and purchased from National cancer institute (NCI), Cairo University, Egypt. The animals were kept under standard environmental conditions on 12 hours light/dark cycle under a constant 
temperature of $(25 \pm 1){ }^{\circ} \mathrm{C}$, and free access to food and water was allowed all the time. An obesity model was performed by feeding with animal tallow, and the effects of this obesity on female rat were studied. Rats under study were randomly divided into nine main equal groups, 10 rats each, placed in individual cages and classified as follows:

\section{Group I: (Control non obese):}

Rats received control normal diet all over the experimental periods (For 18 weeks).

Group П: (Control obese): obese female rats received high fat diet (HFD), served as obesity induced rats group, all over the periods of experiment (For 18 weeks).

Group III: (Starvex): obese female rats received high fat diet (HFD), For 12 weeks then rats were maintained on HFD and received starvex (36 mg/kg b.wt./day/orally) for 6 weeks.

Group IV: (Lupin): obese female rats received high fat diet (HFD) For 12 weeks then rats were maintained on HFD containing 30\% lupin seeds powder for 6 weeks.

Group V: (Purslane): obese female rats received high fat diet (HFD) For 12 weeks then rats were maintained on HFD containing 30\% purslane seeds powder for 6 weeks.

Group VI: (Lupin+Purslane): obese female rats received high fat diet (HFD) For 12 weeks then rats were maintained on HFD containing 30\% lupin and purslane seeds powders for 6 weeks.

Group VII: (Starvex+Lupin): obese female rats received high fat diet (HFD) for 12 weeks then received starvex (36 mg/kg b.wt./day/orally) and maintained on HFD containing 30\% lupin seeds powder for 6 weeks.

Group VIII: (Starvex+Purslane): obese female rats received high fat diet (HFD) for 12 weeks then received starvex $(36 \mathrm{mg} / \mathrm{kg}$ b.wt./day/orally) and maintained on HFD containing $30 \%$ purslane seeds powder for 6 weeks.

Group IX: (Starvex+ Lupin + Purslane): obese female rats received high fat diet (HFD) for 12 weeks then received starvex (36mg/kg b.wt./day/orally) and maintained on HFD containing $30 \%$ purslane and lupin seeds powders for 6 weeks.

\section{Blood samples collection}

Blood was drawn from all animals in each group and centrifuged at $3000 \mathrm{rpm}$ for 10 minutes. Plasma and Serum samples were stored at $0^{\circ} \mathrm{C}$ until biochemical analysis in the same day. Biochemical parameters including, alanine aminotransferase (ALT), aspartate aminotransferase (AST) and lactate dehydrogenase (LDH) as a bio indicators for liver function, blood urea nitrogen (BUN), serum creatinine for kidney functions.

\section{Histology of the Different Tissues for light microscopy}

The animals were sacrificed by cervical dislocation. The liver and kidneys tissue were removed and cutting into small pieces. The specimens were fixed in $10 \%$ neutral formalin solution.

Statistical analysis 
The results were statistically analyzed using analysis of variance (F-test) followed by Duncan's multiple range test to determine differences in means using Statistical Analysis Systems, Version 6.2 (SAS, ${ }^{(14)}$. A p-value less than 0.05 was considered statistically significant.

\section{RESULTS AND DISCUSSION}

Our study was carried out to induce obesity in female rats as an animal model by feeding on high fat diet (HFD) containing $45 \%$ animal fat for 12 weeks. Then, studying the different effects of the obesity and examine the antiobesity effects of oral administration of anti-obesity drug Starvex blue at a dose of $36 \mathrm{mg} / \mathrm{kg}$.b.wt once per day for additional 6 weeks on obese female albino rats feeding on HFD. Also, our study was extended to evaluate the anti-obesity effect of oral administration of lupin and/or purslane seeds powders by feeding obese female rats on HFD mixed with these seeds powder as natural sources of antioxidant once daily for 6 weeks. Finally, to evaluate the possible ameliorative effects of these natural anti-oxidant against any side effects of Starvex.

Serum transaminases (ALT and AST) \& Lactate dehydrogenase (LDH) Regarding the effect of oral administration of Starvex (GPIII) on the serum transaminases (alanine amino transferase (ALT), aspartate amino transferase (AST)) and lactate dehydrogenase (LDH), it is clear that there were highly significant differences in the ALT, AST and LDH activities. Feeding female rats on HFD for 18 weeks (GPII) also induced significant increase ( $<$ < 0.05) in the serum AST, ALT \& LDH activities to higher values than those of the control non obese. However, adding lupin and/or purslane seeds to HFD maintained the serum ALT, AST and LDH activities of obese female rat in groups (IV, V \& VII) to more or less normal control values. But, the higher values of ALT, AST and LDH that above mentioned in Gp III were significantly decreased in female rats treated with the same dose of Starvex to more or less normal values after treated with lupin and/or purslane seeds powders (Figure 1).

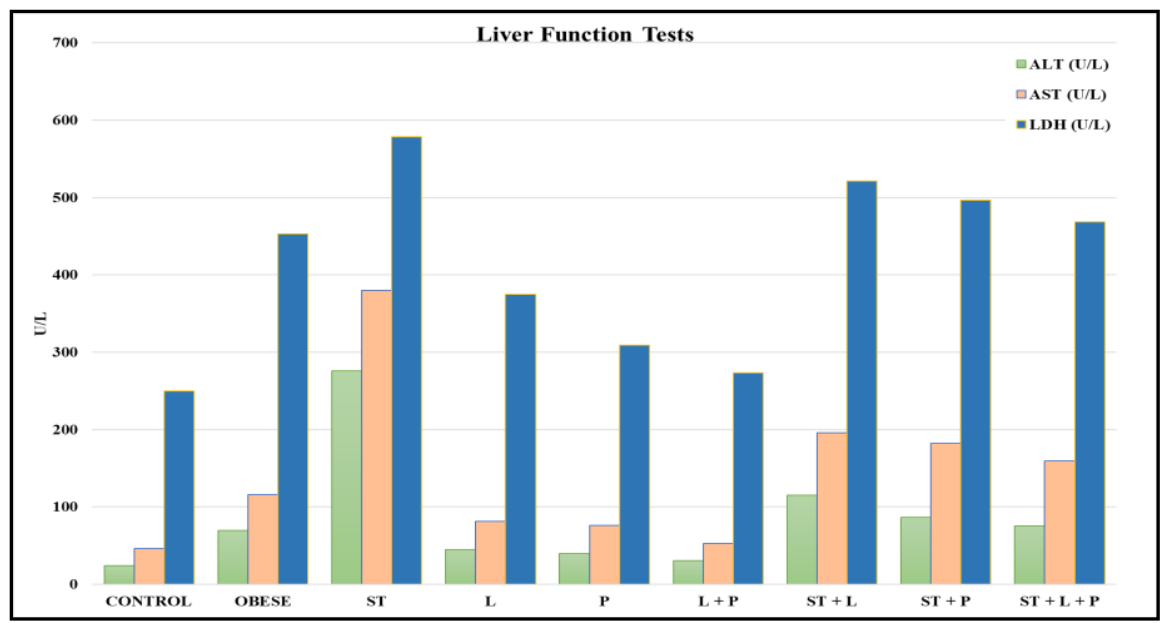

Figure 1: Showing serum liver function tests (ALT, AST \& LDH) in female albino rats treated with Starvex and lupin and/or purslane seeds as antioxidant against side effects of Starvex for 6 weeks. 


\section{Blood urea nitrogen and serum creatinine:}

figures $2 \& 3$ clarify that the levels of BUN and serum creatinine were significantly $(\mathrm{P}<0.05)$ increased in both Starvex treated group (GPIII) and control obesity group (GPII) against normal control group (GPI). The treatment of obese female rats with L in GPIV, $\mathrm{P}$ in GPV and in mixture of $\mathrm{L}+\mathrm{P}$ in GPVI showed significant $(\mathrm{P}<0.05)$ decrease in both BUN level and creatinine when compared to value of female rats fed high fat diet. In contrast, adding lupin and/ or purslane with HFD kept the blood urea nitrogen and serum creatinine to levels of female rat exposed to the same dose of Starvex (GPVII, GPVIII and GPIX) close to values more or less similar to the control obese. So the data showed that the co-treatment of $\mathrm{L}$ and $\mathrm{P}$ in rats fed high fat diet has a renoprotective effect.

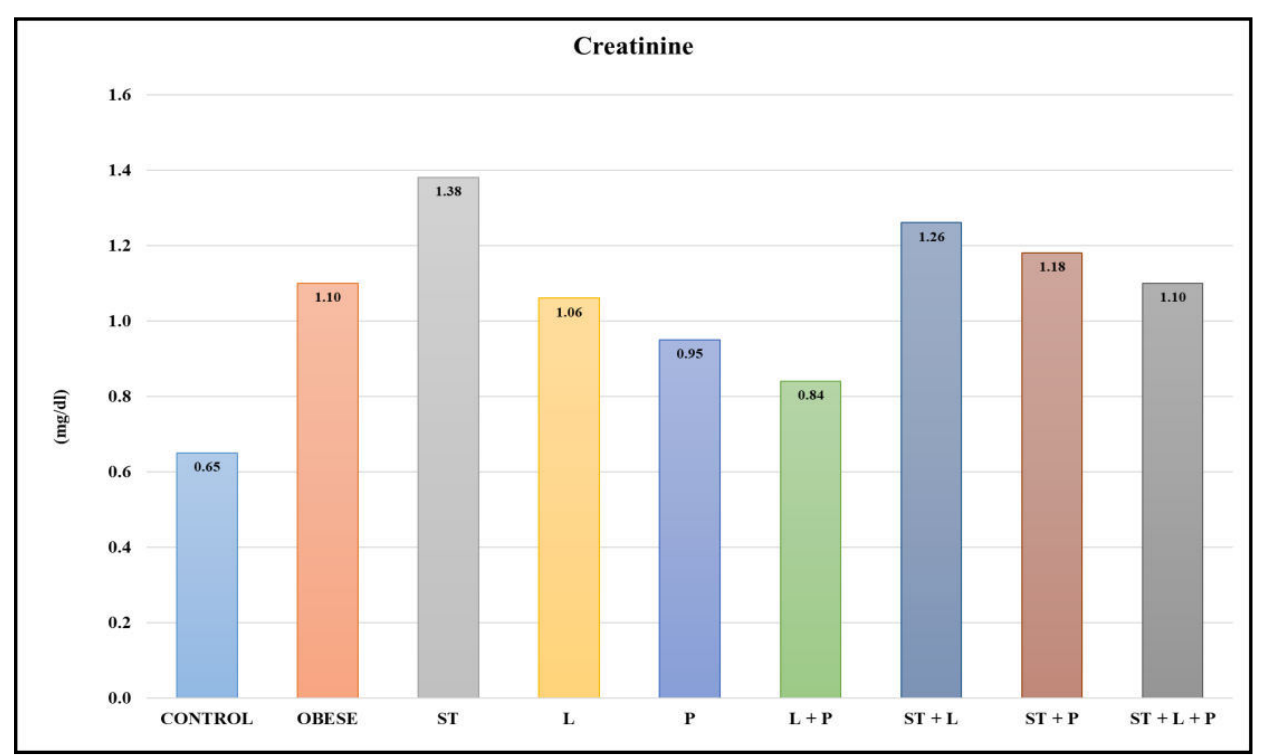

Figure 2: Blood creatinine $(\mathrm{mg} / 100 \mathrm{ml})$ of male albino rats treated with Starvex and lupin and/or purslane seeds as antioxidant against side effects of Starvex for 6 weeks.

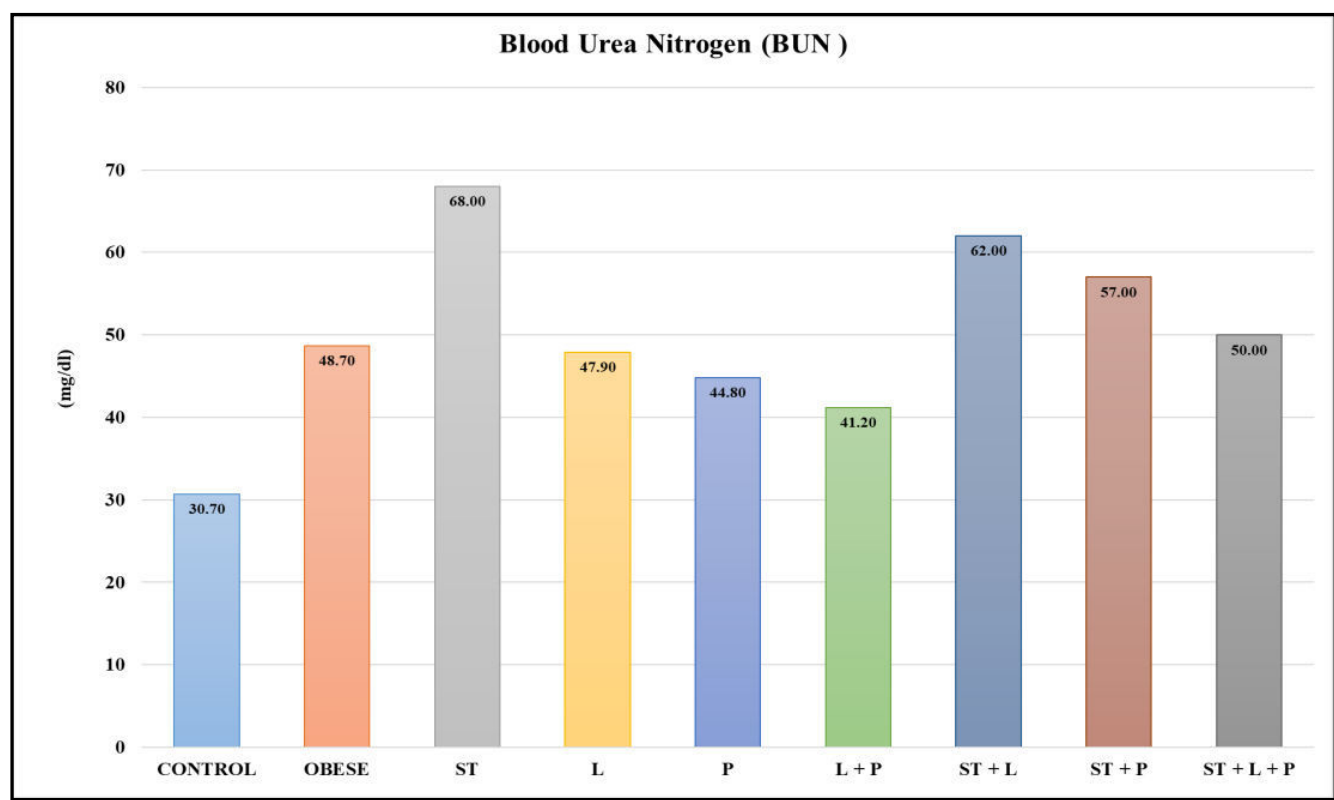

Figure 3: Blood urea nitrogen $(\mathrm{mg} / 100 \mathrm{ml})$ of male albino rats treated with Starvex and lupin and/or purslane seeds as antioxidant against side effects of Starvex for 6 weeks. 


\section{Histopathological observations}

\section{Liver}

Histopathological sections of female rat livers in the different groups were also observed after staining with hematoxylin and eosin (H\&E) (Figure 4). The studied liver parrafin sections revealed that there were no histological abnormalities of hepatocytes in the control non obese group (GPI) with no fat droplets, whereas the hepatocytes in the HFD group possessed serious fat vacuoles, indicating that the rats had developed a high degree of hepatic steatosis induced by HFD. Also, in GPIII (Starvex treated), the studied paraffin section showed degenerated hepatocytes with vaculated cytoplasm and pyknotic nuclei, lymphocytic cell infiltration and necrosis. In contrast, combination HFD with lupin and/or purslane can markedly decrease the fat vacuoles of hepatocytes and the whose hepatocytes are in alignment to varying degrees, similar to those of the control group. This in accordance with the ALT, AST \& LDH values in these groups. Suggesting that lupin and/or purslane play an important role in hepatoprotective function via decreasing the liver indexes enhancing liver function, and relieving fatty liver, which contribute to the anti-obesity effects.

\section{Kidney}

The studied paraffin sections of the kidney of female rat in the different groups after staining with hematoxylin and eosin (H\&E) (Figure 5) showed that there were no histological abnormalities of the kidney cortex in the control non obese group (GPI) consisting of Bowman's capsules with glomeruli and renal tubules (proximal and diatal), whereas in the HFD group serious histopathological lesions such as glomerular shrinkage, degeneration and tubular necrosis were observed (Figure B1\&2). Also, in Starvex treated group (GPIII), the studied paraffin section showed partial and complete degenerated glomeruli, tubular cells with pyknotic nuclei, necrosis of large area with lymphocytic cell infiltration (Fig. C1\&2). On the other hand, mixing HFD with lupin and/or purslane seeds powders can markedly ameliorate the above mentioned kidney abnormalities in both control obese (GPII) or starvex (GPIII) where the kidney structures were more or less similar to those of the control group (Figure B $3,4,5, \mathrm{C} 3,4 \& 5)$. This in accordance with the BUN \& creatinine measurements in these groups. 


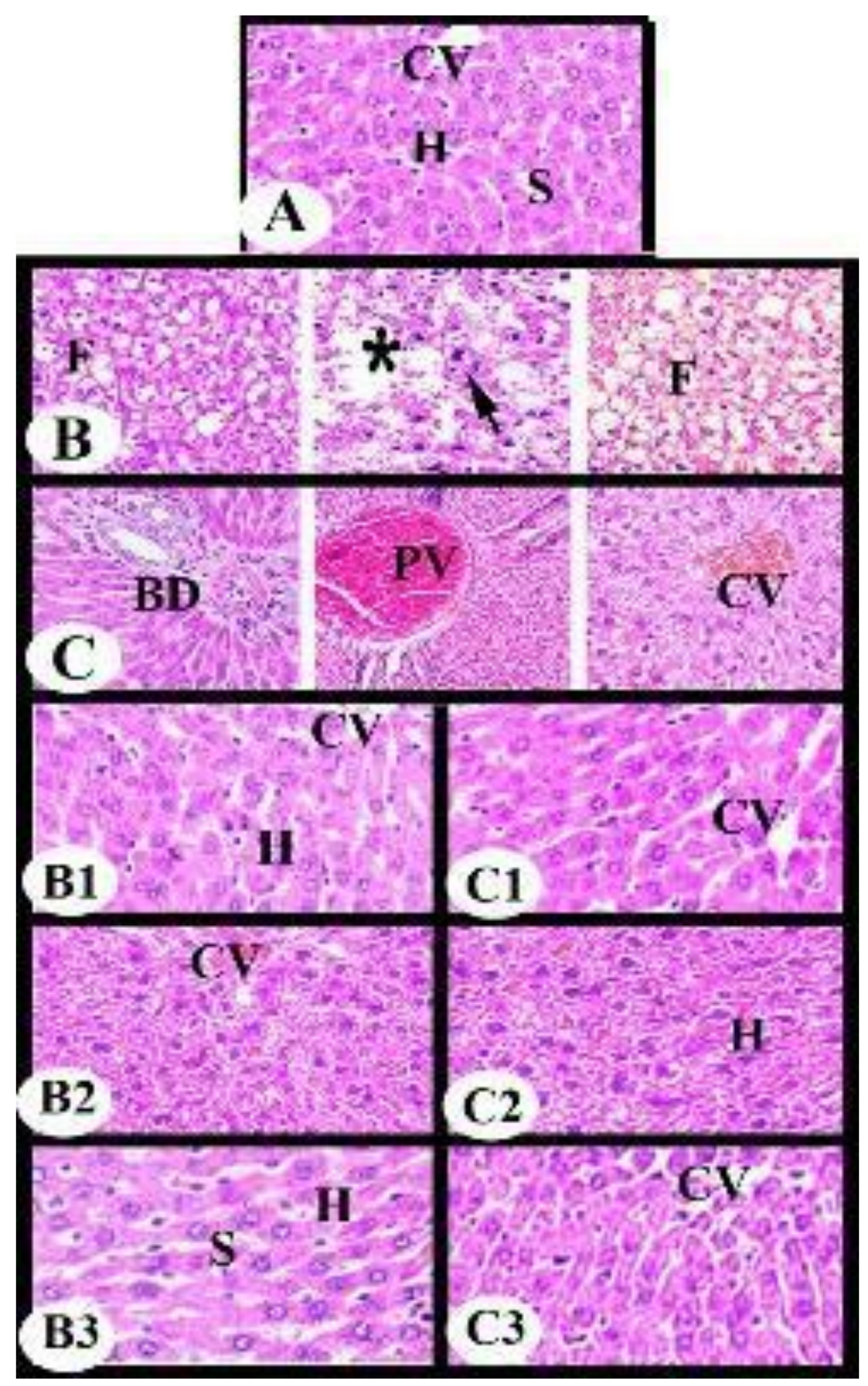

Figure 4: Photomicrographs showing the histological structures of the liver in different groups,

A. Control. B. Obese group. B1. Lupin group. B2. Purslane group. B3. Lupin /Purslane C. Starvex group. C1. Starvex/Lupin group. C2. Starvex/Purslane group. C3. Starvex/lupin/Purslane group. Hepatocyte $(\mathrm{H})$ with vaculated cytopasm $(\mathrm{F})$ or with pyknotic nuclei (arrow), blood sinusoid (S) with kupffer cells $(\mathrm{K})$, large necrotic area (*) with lymphocytic infiltration (L), congested central vein $(\mathrm{CV})$, congested and dilated portal vein

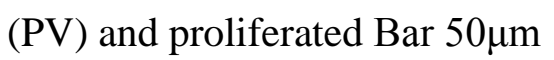




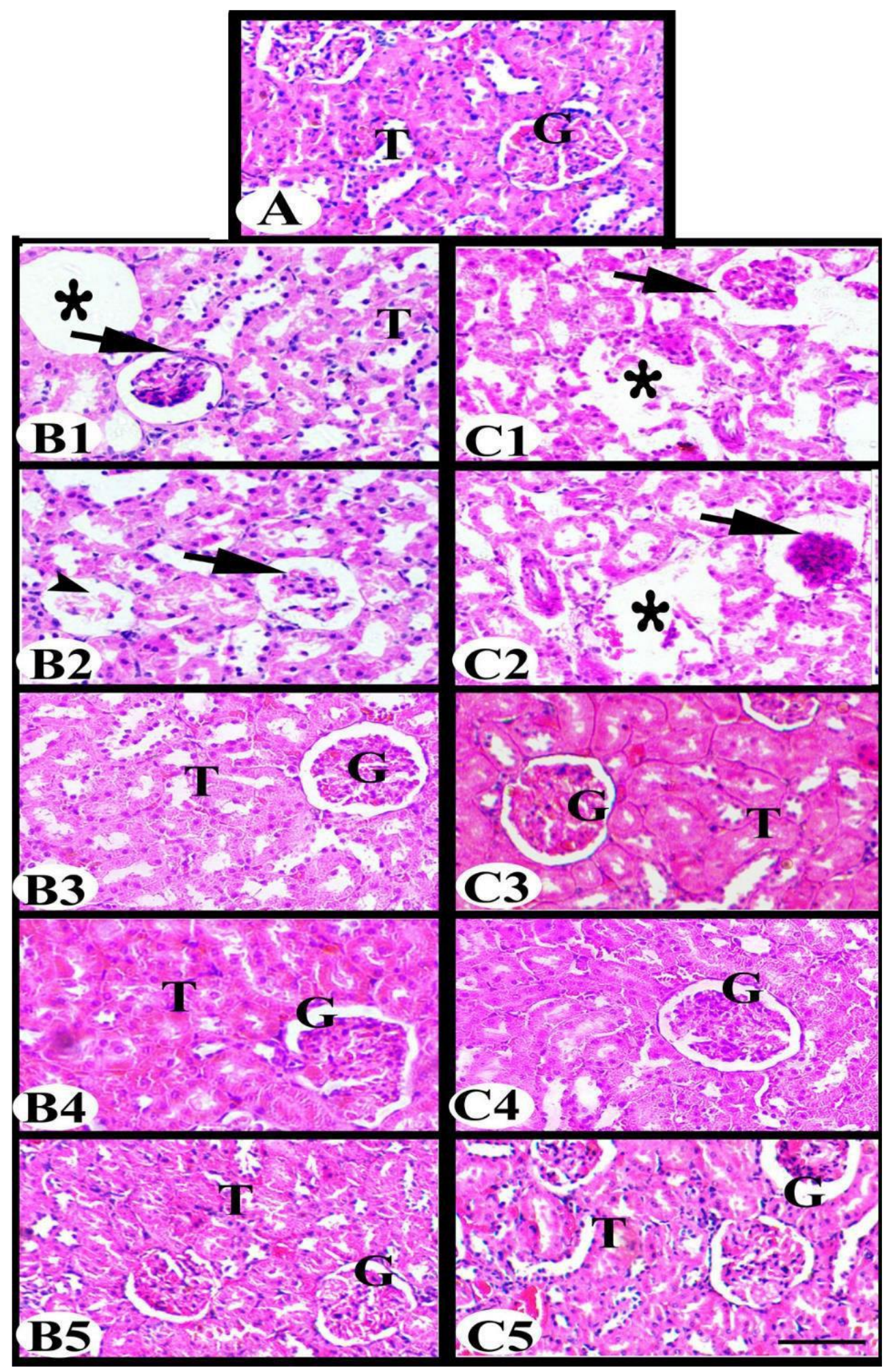

Figure 5: Photomicrographs showing the histological structures of the kidney in different groups,

A. Control. B1\&2. Obese group. B3. Lupin group. B4 Purslane group. B5. Lupin /Purslane C1\&2. Starvex group. C3. Starvex/Lupin group. C4. Starvex/Purslane group. C5. Starvex/lupin/Purslane group. Note: glomerulus (G), shrinkage or cleavage glomerulus

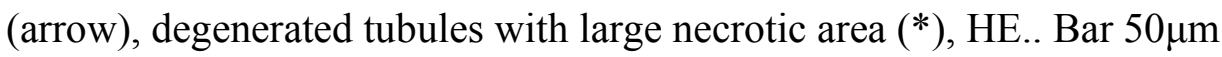




\section{DISCUSSION:}

Obesity is becoming a worldwide epidemic, associated with the increasing risk of metabolic syndrome, diabetes, cardiovascular disease, hypertension and certain cancers. With a global increase in the prevalence of obesity, both nutrition and exercise play key roles in its prevention and treatment (Mir et al., ${ }^{15}$. The World Health Organization estimated that in 2016 more than 1.9 billion adults were overweight (39\% of the population) and over 650 million (13\% of the population) were people with obesity (WHO, ${ }^{16}$. Recently, several anti-obesity drugs approved by the Food and Drug Administration (FDA) have been withdrawn from the market because of their unexpected adverse effects (Onakpoya et al., ${ }^{17}$; Srivastava and Apovian, ${ }^{18}$. Therefore, it is necessary to develop natural products as alternative sources for weight loss agents due to their significant anti-obesity activity, novel structure, and potentially less severe side effects (Pan et al., ${ }^{19}$. Plants have been used in the management of a broad spectrum of metabolic dysfunction including obesity owing to the presence of plant-derived secondary metabolites or natural active components including flavonoids, polyphenols and anthocyanins (Mir et al., ${ }^{15}$. The advantages of using natural preparations are easy availability, better usability, lack of adverse effects, and economic cost-effectiveness (Oršolić et al., ${ }^{20}$.

Some biochemical parameters with some histological examinations of the liver and kidney will be assessed to highlight the effects of lupin individually or in combination with purslane against Starvex toxicity in HFD-induced obese female rat model.

\section{Hepatotoxicity (ALT, AST and LDH) and histopathology}

Liver function tests are important indicators to reveal the functional Status of liver since it is the vital organ involved in detoxification of compounds and in general metabolism. The liver of obese rats displayed characteristic features of hepatic steatosis such as fat accumulation and swelling of rough endoplasmic reticulum and mitochondria in hepatocytes (Guzzaloni et al., ${ }^{21}$; Das et al., ${ }^{22}$.

The current study revealed that all measured liver function tests (ALT, AST \& LDH) were significantly elevated in starvex group (GPIII) comparing with other treated groups. Also, female obese rat showed significant increase in the transaminases and lactate dehydrogenase comparing with other groups. This in accordance with the recorded histopathological results in the liver sections such as in the same groups.

Increased levels of serum ALT, AST and ALP in HFD group, as shown in the present study indicate alterations in liver metabolic function. Additionally, in obesity the fat accumulation in the cytoplasm of fatty hepatocytes caused a leakage of cytoplasmic ALT into the blood. Parallel with our histopathological observations, Altunkaynak ${ }^{23}$ revealed that liver damage including mononuclear cell infiltrations, foci of necrosis, vascular dilatation, an increase in hepatic 
connective tissue, hepatocellular steatosis and shrinkage, additional cytoplasmic acidophily and nuclear density in the hepatocytes of female rat after fat-rich diet (\%30) for 2-3 months. Also, based on the fact that the more damage of the tissue cells is indicative to higher release of these enzymes in blood,

(Liver injury, or hepatotoxicity, is the main relative factor of hyperlipidemia and obesity (Polyzos et al., ${ }^{24}$

The liver is mostly regarded as an essential organ in lipid metabolism. Imbalance between lipid deposition and removal results in hepatic lipid accumulation, which is related to increased hepatic lipogenesis, augmented lipid uptake and/or declined triglyceride export of $\beta$-oxidation (Wang et al., ${ }^{25}$.

In accordance with our results, Pan et al. ${ }^{19}$ revealed that, there were no histological abnormalities of hepatocytes in the normal feeding group with no fat droplets, whereas the hepatocytes in the high fat group possessed serious fat vacuoles, indicating that the rats had developed a high degree of hepatic steatosis induced by HFD. In contrast with ALT, AST and LDH values and the histopathological abnormalities recorded in the hepatocytes of antiobesity starvex treated group, the results achieved by El-Boghdady et al. ${ }^{26}$ found that, antiobesity resveratrol significantly decreased ALT and/or AST activity in animal models (mice or rats) with induced by obesity and Bharrhan et al. ${ }^{27}$ who showed that treatment of HFD-fed rats with resveratrol significantly decreased serum AST, ALT and ALP activities compared to obese non - treated control group. Groups of female obese rats that treated with lupin and/or purslane seeds or in combination with starvex exhibited marked improvement in the values of the liver function enzymes.

So, these natural products may provide hepato-protective effect. In the same line, supplemented hypocholesterolemic diet with $20 \%$ purslane fresh and seed caused significant decrease of activity of AST and ALT compared with hypercholesterolemic group (Shehata and Soltan, ${ }^{28}$. Also, purslane could significantly reduce serum Aspartate Aminotransferase (AST), Alanine and Aminotransferase (ALT) activity (Xiao-xu et al, ${ }^{29}$. Purslane bread was superior in the prevention of hepatic lipid accumulation (Mohamed et al., ${ }^{30}$. The decreased activity of the liver enzymes, ALT, AST, y-GT and ALP in purslane treated group were possibly due to the antioxidants contents (vitamins, a-tocopherol, ascorbic acid and 3- carotene, as well as glutathione) that act against oxidative stress, indicates its protective role against liver damage (Hussein $^{31}$; Dkhil et al., ${ }^{32}$.

The protective action might be due to the potent antioxidant property of purslane seeds or due to its action against enhancement of hepatic steatosis and fat accumulation in liver (Hussein, ${ }^{31}$; Dkhil et al., ${ }^{32}$; EI-Serwy and Abd, ${ }^{33}$. Purslane seed contained the highest level of polyunsaturated fatty acids (omega-3 fatty acid) (Shehata and Soltan, ${ }^{28}$. The melatonin 
concentration in purslane was found to exceed that reported in a number of other fruits and vegetables (Simopoulos et al. ${ }^{34}$. Melatonin has a variety of important functions including direct free radical scavenging and anti-inflammatory properties (Rodriguez et al. ${ }^{35}$. The obese rats on the high-protein diet also exhibited lower liver weights and less fat deposition in the liver, compared to those receiving the moderate-protein diet (French et al., ${ }^{36}$. Similar with our findings, ethanolic extract of Terminalia paniculata (TPEE) administration has effectively lowered the HFD induced elevated levels of these hepatic enzymes and lipid profiles demonstrating its protective activity. (Ramgopal et al., ${ }^{37}$. Also, chitosan oligosaccharide capsules, especially high dose (COSC-H) can markedly decrease the fat vacuoles of hepatocytes whose cells are in alignment to varying degrees, similar to those of the normal feeding group.

The increased serum AST and ALT were shown in the high fat (HF) group when compared to that in the normal diet group (NF) were significantly lowered to the normal range. In particular, the effects of high and medium dose of chitosan oligosaccharide capsules (COSC) were superior to those of Orlistat $(\mathrm{p}<0.05)$, suggesting that COSCs play an important role in hepatoprotective function (Pan et al., ${ }^{19}$.

\section{Nephrotoxicity:}

Our results showed that kidney function tests (BUN \& creatinine) were significantly elevated in both starvex and obese groups (GPIII \& GPII) in comparing with control non obese and other treated groups. This in agreement with the recorded histopathological lesions such as glomerular shrinkage and degeneration, degenerated tubules, large necrotic areas in both obese and starvex treated groups. But, most of these lesions were repaired up after lupin and or purslane treatments and this is in agreement with our improved kidney function tests. These results are in agreement with, Lewis et al. ${ }^{38}$ who reported that, cafeteria diet fed rats showed significant increase in serum creatinine, uric acid levels and decreased blood urea levels.

Serum levels of BUN are used to determine kidney damage. Moreover, our findings are consistent with previous reports showing that high fat-dependent obesity is associated with many kidney alterations (Jiang et al., ${ }^{39}$; Altunkaynak et al., ${ }^{40}$. Also, chronic administration of dietetic lipid can cause abdominal obesity and can significantly alter the renal cortical structure of rats (Aguila \& Mandarim-De-Lacerda, ${ }^{41}$. Moreover, the results of Yurt et al ${ }^{42}$ study suggest that there is a significant relationship between fatty diet intake and structural changes of the kidney. Expanded Bowman space, irregular urinary space, and decrease in the numerical density of glomeruli, glomerular necrosis and atrophy, basal membrane thickening, mononuclear cell infiltration, fibrin accumulation, hydropic degeneration, segmental necrosis prominent dilatation of the renal vessels. Several mechanisms may contribute to the onset and/or the progression of renal involvement in experimental obesity among them; lipid 
peroxidation and oxidative stress have been frequently proposed. HFD induces alteration of renal lipid metabolism by an imbalance between lipogenesis and lipolysis in the kidney, as well as systemic metabolic abnormalities and subsequent renal lipid accumulation and lipid peroxidation leading to renal injury (Kume et al., ${ }^{43}$.

A study by Dkhill et al. ${ }^{32}$ showed that purslane administration at $1.5 \mathrm{mg} / \mathrm{kg}$ purslane aqueous juice for 12 days caused significant decrease in urea and creatinine respectively. Also, Barakat and Mahmoud ${ }^{(44)}$ proved the efficiency of using either flax/pumpkin or purslane/pumpkin seed mixture (components of $\omega-3$ and $\omega 6$ ) on kidney function in rats fed high cholesterol diets. Treatment with starvex, lupin and/or purslane individually and in combination inhibited the proliferation and size of adipocytes, as fewer and smaller adipocytes were observed compared to those in the high fat group, using the same magnification and field of view. Therefore, the studied materials can effectively exert anti-obesity effects by suppressing the growth and accumulation of adipocytes. Similar results were reported by Pan et al., ${ }^{19 \mathrm{~s}}$.

\section{CONCLUSION}

Starvex, is one of the drugs used in the treatment of obesity. So usage of the antioxidant in concomitant with the drug is mandatory. On the other hand, purslane and lupin are two of the most natural materials used

\section{REFERENCES}

1. Sharpe, P. A., Blanck, H. M., Williams, J. E., Ainsworth, B. E., \& Conway, J. M. (2007). Use of complementary and alternative medicine for weight control in the United States. The Journal of Alternative and Complementary Medicine, 13(2), 217-222.

2. Ebrahimzadeh Attari, V., Mahdavi, A. M., Javadivala, Z., Mahluji, S., Vahed,S. Z., \& Ostadrahimi, A. (2018). A systematic review of the antiobesity and weight lowering effect of ginger (Zingiber officinale Roscoe) and its mechanisms of action. Phytotherapy Research, 32, 577-585.

3. WHO (2015). World Health Organization. Obesity and overweight (Online).[Cited 2015]; Available from: URL: http://www.who.int/mediacentre/factsheets/fs311/en

4. Cheung, B. M. Y., Cheung, T. T., \& Samaranayake, N. R. (2013). Safety of antiobesity drugs. Therapeutic advances in drug safety, 4(4), 171-181.

5. Habibuddin M., \& Humaira, T. (2014). Pharmacological management of obesity: Past, present and future. Saudi Journal of Obesity, 2(1), Page: 3- 12.

6. Ekor, M. (2014). The growing use of herbal medicines: issues relating to adverse reactions and challenges in monitoring safety. Frontiers in pharmacology, 4, 177. 
7. Fu, C., Jiang, Y., Guo, J., \& Su, Z. (2016). Natural products with anti-obesity effects and different mechanisms of action. Journal of agricultural and food chemistry, 64(51), 9571-9585.

8. Bhutani, K. K., Birari, R., \& Kapat, K. (2007). Potential anti-obesity and lipid lowering natural products: a review. Natural Product Communications, 2(3), $1934578 X 0700200316$.

9. Yang, L., Chen, J. H., Lv, J., Wu, Q., Xu, T., Zhang, H., ... \& Yang, H. K(2012). Rice protein improves adiposity, body weight and reduces lipids level in rats through modification of triglyceride metabolism. Lipids in health and disease, 11(1), 24.

10. Elbandy, M., \& Rho, J. R. (2014). New flavone-di-C-glycosides from the seeds of Egyptian lupin (Lupinus termis). Phytochemistry Letters, 9, 127-131.

11. El-Zawahry, F., Morsey, D., Farrag , M., Mustafa, M., Al-Gebily, K., Abdel-Wahhab E. (2019). Egyptian Purslane (Portulaca oleracea L.) efficacy on amelioration of BMI, PON1 and Irisin in obese rats. International Journal of Biology Research Vol. 4; Issue 1; Jan; Page No. 46-54

12. Iossa, Susanna, Lillà Lionetti, Maria Pina Mollica, Antonio Barletta, and Giovanna Liverini. (1999). "Energy intake and utilization vary during development in rats.” The Journal of nutrition 129, no. 8, 1593-1596.

13. Preston, S. H., Mehta, N. K., \& Stokes, A. (2013). Modeling obesity histories in cohort analyses of health and mortality. Epidemiology (Cambridge, Mass.), 24(1)

14. SAS Institute. (2000). JMP: Statistics and Graphics Guide. Sas Inst.

15. Mir, S. A., Shah, M. A., Ganai, S. A., Ahmad, T., \& Gani, M. (2019). Understanding the role of active components from plant sources in obesity management. Journal of the Saudi Society of Agricultural Sciences, 18(2), 168-176.

16. WHO (2018). World Health Organization. Fact sheet obesity and over weight's 2018. http://www.who.int/news-room/fact sheets/detail/obesity-and over w eight.

17. Onakpoya, I. J., Heneghan, C. J., \& Aronson, J. K. (2016). Post-marketing withdrawal of anti-obesity medicinal products because of adverse drug reactions: a systematic review. BMC medicine, 14(1), 191.

18. Srivastava, G., \& Apovian, C. (2018). Future pharmacotherapy for obesity: new antiobesity drugs on the horizon. Current obesity reports, 7(2), 147-161.

19. Pan, H., Fu, C., Huang, L., Jiang, Y., Deng, X., Guo, J., \& Su, Z. (2018). Antiobesity effect of chitosan oligosaccharide capsules (COSCs) in obese rats by ameliorating leptin resistance and adipogenesis. Marine drugs, 16(6), 198. 
20. Oršolić, N., Landeka Jurčević, I., Đikić, D., Rogić, D., Odeh, D., Balta, V., \& Jutrić, D. (2019). Effect of propolis on diet-induced hyperlipidemia and atherogenic indices in mice. Antioxidants, 8(6), 156.

21. Guzzaloni, G., Grugni, G., Minocci, A., Moro, D., \& Morabito, F. (2000). Liver steatosis in juvenile obesity: correlations with lipid profile, hepatic biochemical parameters and glycemic and insulinemic responses to an oral glucose tolerance test. International journal of obesity, 24(6), 772- 776.

22. Das N, Sikder K, Ghosh S, Fromenty B, Dey S. Moringa oleifera Lam. leaf extract prevents early liver injury and restores antioxidant status in mice fed with high-fat diet.

23. Altunkaynak, Z. (2005). Effects of high fat diet induced obesity on female rat livers (a histochemical study). Eur J Gen Med 2005; 2(3):100-109

24. Polyzos, S. A., Kountouras, J., \& Mantzoros, C. S. (2017). Adipose tissue, obesity and non-alcoholic fatty liver disease. Minerva endocrinologica, 42(2), 92-108.

25. Wang, L. F., Wang, X. N., Huang, C. C., Hu, L., Xiao, Y. F., Guan, X. H., ... \& Xin, H. B. (2017). Inhibition of NAMPT aggravates high fat diet induced hepatic steatosis in mice through regulating Sirt1/AMPK $\alpha / \mathrm{SREBP1}$ signaling pathway. Lipids in health and disease, $16(1), 82$.

26. El-Boghdady NA, Abdeltawab NF, Nooh MM. Resveratrol and Montelukast alleviate paraquat-induced hepatic injury in mice: modulation of oxidative stress, inflammation, and apoptosis. Oxidative medicine and cellular longevity. 2017 Jan 1;2017.

27. Bharrhan, S., Chopra, K., \& Rishi, P. (2010). Vitamin E supplementation modulates endotoxin-induced liver damage in a rat model. Am J Biomed Sci, 2(1), 51-62.

28. Shehata MM, Soltan SS. The effects of purslane and celery on hypercholesterolemic mice. World Journal of Dairy \& Food Sciences. 2012;7(2):212-21.

29. Xiao-Xu H, YA-Wei 1, Rong-Chao Z, Qing-Shan, Chun-mei S (2011). Protective effect of Purslane on hyperlipidemic rat livers. Journal of Jilin Medical College, 32(1):16732995.

30. Mohamed, D. A., Abdelgayed, S. S., Essa, H. A., \& Mohamed, R. S. (2018). Preparation and Evaluation of Functional Foods for Prevention of Nonalcoholic Fatty Liver Disease. Sciences, 21(9), 454-462.

31. Hussein, M. A. (2010). Purslane extract effects on obesity-induced diabetic rats fed a high-fat diet. Malaysian journal of nutrition, 16(3).

32. Dkhil, M. A., Moniem, A. A., Al-Quraishy, S., \& Saleh, R. A. (2011). Antioxidant effect of purslane (Portulaca oleracea) and its mechanism of action. J Med Plants Res, 5(9), 1589-1563. 
33. EI-Serwy, E., \& Abd, E. I. (2012). Influence of Sage (Salvia officinalis L.) and Purslane (Portulaca oleracea L.) on Weight Reduction and Some Biochemical Parameters in Rats Suffering from Obesity. Egyptian Journal of Nutrition and Health, 7(1), 1-16.

34. Simopoulos, A. P., Tan, D. X., Manchester, L. C., \& Reiter, R. J. (2005). Purslane: a plant source of omega-3 fatty acids and melatonin. Journal of Pineal Research, 39(3), $331-332$.

35. Rodriguez, C., Mayo, J. C., Sainz, R. M., Antolín, I., Herrera, F., Martín, V., \& Reiter, R. J. (2004). Regulation of antioxidant enzymes: a significant role for melatonin. Journal of pineal research, 36(1), 1-9.

36. French, W. W., Dridi, S., Shouse, S. A., Wu, H., Hawley, A., Lee, S. O., ... \& Baum, J. I. (2017). A high-protein diet reduces weight gain, decreases food intake, decreases liver fat deposition, and improves markers of muscle metabolism in obese Zucker rats. Nutrients, 9(6), 587.

37. Ramgopal, M., Kruthika, B. S., Surekha, D., \& Meriga, B. (2014). Terminalia paniculata bark extract attenuates non-alcoholic fatty liver via down regulation of fatty acid synthase in high fat diet-fed obese rats. Lipids in health and disease, 13(1), 58.

38. Lewis, A. R., Singh, S., \& Youssef, F. F. (2019). Cafeteria-diet induced obesity results in impaired cognitive functioning in a rodent model. Heliyon, 5(3), e01412.

39. Jiang, T., Wang, Z., Proctor, G., Moskowitz, S., Liebman, S. E., Rogers, T., ... \& Levi, M. (2005). Diet-induced obesity in C57BL/6J mice causes increased renal lipid accumulation and glomerulosclerosis via a sterol regulatory element-binding protein1c-dependent pathway. Journal of biological chemistry, 280(37), 32317-32325.

40. Altunkaynak, M. E., Özbek, E., Altunkaynak, B. Z., Can, İ., Unal, D., \& Unal, B. (2008). The effects of high-fat diet on the renal structure and morphometric parametric of kidneys in rats. Journal of anatomy, 212(6), 845-852.

41. Aguila, M. B., \& Mandarim-de-Lacerda, C. A. (2003). Heart and blood pressure adaptations in Wistar rats fed with different high-fat diets for 18 months. Nutrition, 19(4), 347-352.

42. Yurt, K. K., Kayhan, E., Altunkaynak, B. Z., Tümentemur, G., \& Kaplan, S. (2013). Effects of the melatonin on the kidney of high fat diet fed obese rats: A stereological and histological approach. Journal of experimental and clinical medicine, 30(2), 153158.

43. Kume, S., Uzu, T., Araki, S. I., Sugimoto, T., Isshiki, K., Chin-Kanasaki, M., \& Haneda, M. (2007). Role of altered renal lipid metabolism in the development of renal injury induced by a high-fat diet. Journal of the American Society of Nephrology, 18(10), 2715-2723. 
44. Barakat, L. A., \& Mahmoud, R. H. (2011). The antiatherogenic, renal protective and immunomodulatory effects of purslane, pumpkin and flax seeds on hypercholesterolemic rats. North American journal of medical sciences, 3(9), 411.

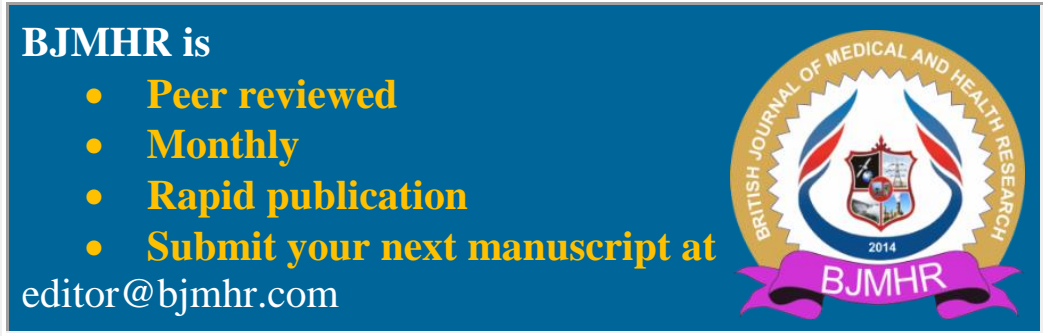

